\title{
Applications of variational methods to the impulsive equation with non-separated periodic boundary conditions
}

Ruixi Liang and Wei Zhang*

"Correspondence:

weizhangcsu@sina.com Department of Mathematics and Statistic, Central South University Changsha, Hunan 410073, China

\begin{abstract}
In this paper, we study the existence of classical solutions for a second-order impulsive differential equation with non-separated periodic boundary conditions. By using the variational method and critical point theory, we give some new criteria to guarantee that the impulsive problem has at least one solution under some different conditions. Our results extend and improve some recent results.
\end{abstract}

MSC: $34 \mathrm{~B} 37 ; 37 J 45$

Keywords: impulsive differential equations; critical point theory; non-separated periodic boundary conditions

\section{Introduction}

Many dynamical systems have an impulsive dynamical behavior due to abrupt changes at certain instants during the evolution process. The mathematical description of these phenomena leads to the impulsive differential equations. Recent developments in this field have been motivated by many applied problems, such as control theory [1, 2], population dynamic [3], medicine [4-6], and some physics or mechanics problems [7]. In the last few years, a great deal of work has been done in the study of the existence of solutions for impulsive boundary value problems. Some classical tools or techniques have been used to study such problems in the literature, such as coincidence degree theory of Mawhin [8], the method of upper and lower solutions with the monotone iterative technique [9-12], and some fixed point theorems in cones [13-15]. For some general and recent work on the theory of impulsive differential equation, we refer the interested reader to [16-18].

In this paper, we are concerned with the existence of solutions for the following boundary value problem (BVP) with impulses:

$$
\begin{aligned}
& -\left(p(t) u^{\prime}(t)\right)^{\prime}=f(t, u(t)), \quad t \neq t_{k}, t \in J, \\
& \Delta\left(u^{[1]}\left(t_{k}\right)\right)=I_{k}\left(u\left(t_{k}\right)\right), \quad k=1,2, \ldots, m, \\
& u(0)=u(T), \quad u^{[1]}(0)=u^{[1]}(T) .
\end{aligned}
$$

Here $T$ be a fixed positive number, $u^{[1]}(t)=p(t) u^{\prime}(t)$ denotes the quasi-derivative of $u(t)$. Condition (1.1c) is called a non-separated periodic boundary value condition for (1.1a).

(C) 2016 Liang and Zhang. This article is distributed under the terms of the Creative Commons Attribution 4.0 International License (http://creativecommons.org/licenses/by/4.0/), which permits unrestricted use, distribution, and reproduction in any medium, provided you give appropriate credit to the original author(s) and the source, provide a link to the Creative Commons license, and indicate if changes were made. 
We assume throughout, and without further mention, that the following conditions hold.

(H) Let $J=[0, T]$, and $0=t_{0}<t_{1}<t_{2}<\cdots<t_{m}<t_{m+1}=T, f \in C\left(J \times \mathbb{R}^{+}, \mathbb{R}^{+}\right), I_{k} \in C(\mathbb{R}, \mathbb{R})$, $\mathbb{R}^{+}=[0,+\infty) . \Delta\left(u^{[1]}\left(t_{k}\right)\right)=u^{[1]}\left(t_{k}^{+}\right)-u^{[1]}\left(t_{k}^{-}\right)$, where $u^{[1]}\left(t_{k}^{+}\right)$(respectively, $\left.u^{[1]}\left(t_{k}^{-}\right)\right)$denotes the right limit (respectively left limit) of $u^{[1]}(t)$ at $t=t_{k}$.

(H1) The function $f(t, u)$ is measurable in $t \in[0, T]$ for each $u \in \mathbb{R}$, continuous in $u \in \mathbb{R}$ for a.e. $t \in[0, T]$ (Caratheodory function), and there exist $a(t) \in C\left(\mathbb{R}^{+}, \mathbb{R}^{+}\right)$and a Lebesgue measurable function $b(t) \geq 0$ such that $b(t) \in L^{1}\left(0, T ; \mathbb{R}^{+}\right)$and $|f(t, u)| \leq a(|u|) b(t)$, for all $u \in \mathbb{R}$ and a.e. $t \in[0, T]$. We have

$$
\int_{0}^{T} \frac{1}{p(t)} d t<\infty, \quad p(t)>0 \text { on }[0, T]
$$

A function $u(t)$ defined on $J^{-}=J \backslash\left\{t_{1}, t_{2}, \ldots, t_{m}\right\}$ is called a classical solution of BVP (1.1a)(1.1c) if its first derivative $u^{\prime}(t)$ exists for each $t \in J^{-}, p(t) u^{\prime}(t)$ is absolutely continuous on each closed subinterval of $J^{-}$, there exist finite values $u^{[1]}\left(t_{k}^{ \pm}\right)$, the impulse conditions $(1.1 \mathrm{~b})$, and the boundary conditions (1.1c) are satisfied, and equation (1.1a) is satisfied almost everywhere on $J^{-}$.

For the case of $I_{k}=0(k=1,2, \ldots, m)$, problem (1.1a)-(1.1c) is related to a non-separated periodic boundary value problem of ODE. More precisely, Atici and Guseinov [19] considered the following non-separated periodic boundary value problem:

$$
\left\{\begin{array}{l}
-\left[p(x) y^{\prime}\right]^{\prime}+q(x) y=f(x, y), \quad 0 \leq x \leq \omega \\
y(0)=y(\omega), \quad y^{[1]}(0)=y^{[1]}(\omega) .
\end{array}\right.
$$

The authors proved the existence of a positive and twin positive solutions to BVP (1.2) by applying a fixed point theorem for the completely continuous operators in cones.

Based upon the properties of the Green's function obtained in [19], Graef and Kong [20] extended and improved the work of [19] by using topological degree theory. They derived new criteria for the existence of non-trivial solutions, positive solutions, and negative solutions of problem (1.2) when $f$ is a sign-changing function and not necessarily bounded from below even over $[0, \omega] \times \mathbb{R}^{+}$.

Very recently, by introducing a variational framework for a class of second-order nonlinear differential equations with non-separated periodic boundary value conditions, Han [21] obtained some results on the existence of non-trivial, positive, and negative solutions of problem (1.2), where the nonlinearities are unbounded and satisfy Ahmad-Lazer-Paul type conditions. The problem (1.2) in the case of $p \equiv 1$, the usual periodic boundary value problem, has been extensively investigated; see $[22,23]$ for some results.

For the impulsive case of $(1.2)$, Huseynov $[24,25]$ considered the following problem:

$$
\left\{\begin{array}{l}
-\left[p(x) y^{\prime}(x)\right]^{\prime}+q(x) y(x)=h(x), \quad x \in[a, c) \cup(c, b], \\
y\left(c^{-}\right)=d_{1} y\left(c^{+}\right), \quad y^{[1]}\left(c^{-}\right)=d_{2} y^{[1]}\left(c^{+}\right), \\
y(a)=y(b), \quad y^{[1]}(a)=y^{[1]}(b) .
\end{array}\right.
$$

In [24], Huseynov investigated the Green's function of the boundary value problem (1.3), sufficient conditions that ensure the positiveness of the Green's function are established. The author also investigated nonlinear second-order differential equations subject to linear impulse conditions and non-separated periodic boundary conditions in [25]. 
Motivated by the above facts, in this paper, our aim is to study the existence of solutions for the impulsive boundary value problem (1.1a)-(1.1c). To the best of our knowledge, there has so far been no paper concerning the non-separated boundary value problem with impulses via variational methods. In addition, this paper is a generalization of [21], in which impulsive effects are not involved. For some general and recent work on the critical point theory and variational methods, we refer the reader to [26-36]. It is a novel approach to apply variational methods to the impulsive boundary value problem.

Throughout this paper, we will use the following notations: $L^{q}(0, T)$ is the usual Banach space with norm $\|u\|_{q}=\left(\int_{0}^{T}|u|^{q} d t\right)^{1 / q},\|u\|_{\infty}=\max _{t \in[0, T]}|u(t)|$, and $\|u\|_{p, 2}=\left(\int_{0}^{T} p u^{2} d t\right)^{1 / 2}$. $C, C_{1}, C_{2}, \ldots$ denote the positive (possibly different) constants.

\section{Preliminaries}

In this sections, we recall some basic facts which will be used in the proofs of our main results. In order to apply the critical point theory, we construct a variational structure. With this variational structure, we can reduce the problem of finding solutions of (1.1a)(1.1c) to that of seeking the critical points of a corresponding functional.

Let $W_{p, T}^{1,2}$ be the Sobolev space

$$
W_{p, T}^{1,2}=\left\{u \in A C[0, T] \mid u(0)=u(T), \sqrt{p} u^{\prime} \in L^{2}(0, T)\right\}
$$

with the norm

$$
\|u\|=\left(\int_{0}^{T} p(t)\left|u^{\prime}(t)\right|^{2} d t+\int_{0}^{T} u^{2}(t) d t\right)^{1 / 2} .
$$

Certainly, $W_{p, T}^{1,2}$ is also a Hilbert space with the inner product induced by its norm. For more details, see [21].

In the meantime, according to Proposition 2.5 in [21], the problem

$$
\left\{\begin{array}{l}
-\left(p(t) u^{\prime}(t)\right)^{\prime}=\mu u, \\
u(0)=u(T), \quad u^{[1]}(0)=u^{[1]}(T)
\end{array}\right.
$$

has a sequence of eigenvalues, $0=\mu_{0}<\mu_{1}<\cdots<\mu_{n}<\cdots$, and corresponding eigenfunctions, $\psi_{n}(n=0,1,2, \ldots)$, where we can choose $\psi_{0}(t) \equiv 1$ in $[0, T]$. It is obvious that $\psi_{n}(n=0,1, \ldots)$ are mutually orthogonal in $L^{2}(0, T)$. Set $\bar{W}=\operatorname{span}\{1\} \subset W_{p, T}^{1,2}$ and $\widetilde{W}=$ $\overline{\operatorname{span}}\left\{\psi_{1}, \psi_{2}, \ldots\right\} \subset W_{p, T}^{1,2}$, then $W_{p, T}^{1,2}=\bar{W} \oplus \widetilde{W}$. Accordingly, for every $u \in W_{p ; T}^{1,2}, u=\bar{u}+\widetilde{u}$. As in the proof of Proposition 2.5 in [21], we have

$$
\mu_{1} \int_{0}^{T} u^{2} d t \leq \int_{0}^{T} p\left|u^{\prime}\right|^{2} d t, \quad \forall u \in \widetilde{W}
$$

From Theorem 4.1 in [21], we have the inequality

$$
\|\widetilde{u}\|_{\infty} \leq C\left(\int_{0}^{T} p \widetilde{u}^{\prime 2} d t\right)^{1 / 2}, \quad \forall u \in W_{p, T}^{1,2}
$$

The above two important inequalities can be simply rewritten as follows:

$$
\|\widetilde{u}\|_{\infty} \leq C\left\|\widetilde{u}^{\prime}\right\|_{p, 2}, \quad \forall u \in W_{p, T}^{1,2}
$$




$$
\|\widetilde{u}\|_{2}^{2} \leq \frac{1}{\mu_{1}}\left\|\widetilde{u}^{\prime}\right\|_{p, 2}^{2}, \quad \forall u \in \widetilde{W}
$$

Proposition 2.1 ([21], Proposition 2.1) Suppose that the condition (H1) holds, then $W_{p, T}^{1,2}$ is compactly imbedded in $C[0, T]$.

For $u \in W_{p, T}^{1,2}$, we set

$$
J(u)=\frac{1}{2} \int_{0}^{T} p u^{\prime 2} d t-\int_{0}^{T} F(t, u) d t+\sum_{k=1}^{m} \int_{0}^{u\left(t_{k}\right)} I_{k}(s) d s,
$$

where

$$
F(t, u)=\int_{0}^{u} f(t, s) d s
$$

In view of the condition (H1), we can prove that $J(u)$ is well defined and is $C^{1}$ on $W_{p, T}^{1,2}$. Furthermore, we have

$$
\left(J^{\prime}(u), v\right)=\int_{0}^{T} p u^{\prime} v^{\prime} d t-\int_{0}^{T} f(t, u) v d t+\sum_{k=1}^{m} I_{k}\left(u\left(t_{k}\right)\right) v\left(t_{k}\right),
$$

for all $u, v \in W_{p, T}^{1,2}$. The proofs can be given as in the corresponding results in [32,33].

Definition 2.1 $u \in W_{p, T}^{1,2}$ is a weak solution of problem (1.1a)-(1.1c), if

$$
\int_{0}^{T} p u^{\prime} v^{\prime} d t-\int_{0}^{T} f(t, u) v d t+\sum_{k=1}^{m} I_{k}\left(u\left(t_{k}\right)\right) v\left(t_{k}\right)=0,
$$

for all $v \in W_{p, T}^{1,2}$.

By Definition 2.1, the weak solutions of problems (1.1a)-(1.1c) correspond to the critical points of $J$.

Theorem 2.1 If $u \in W_{p, T}^{1,2}$ is a critical point of the functional J, then $u$ is a classical solution of (1.1a)-(1.1c).

Proof Let $u \in W_{p, T}^{1,2}$ be a critical point of the functional $J$. It shows that

$$
\int_{0}^{T} p u^{\prime} v^{\prime} d t-\int_{0}^{T} f(t, u) d t+\sum_{k=1}^{m} I_{k}\left(u\left(t_{k}\right)\right) v\left(t_{k}\right)=0
$$

holds for any $v \in W_{p, T}^{1,2}$. By integrating by parts, we have

$$
\begin{aligned}
0 & =\sum_{k=0}^{m} \int_{t_{k}}^{t_{k+1}} p u^{\prime} v^{\prime} d t-\int_{0}^{T} f(t, u) v d t+\sum_{k=1}^{m} I_{k}\left(u\left(t_{k}\right)\right) v\left(t_{k}\right) \\
& =\sum_{k=0}^{m}\left[\left.p u^{\prime} v\right|_{t_{k}^{+}} ^{t_{k+1}}\right]-\int_{0}^{T}\left(p u^{\prime}\right)^{\prime} v d t-\int_{0}^{T} f(t, u) v d t+\sum_{k=1}^{m} I_{k}\left(u\left(t_{k}\right)\right) v\left(t_{k}\right)
\end{aligned}
$$




$$
\begin{aligned}
= & p\left(t_{1}\right) u^{\prime}\left(t_{1}\right) v\left(t_{1}\right)-p(0) u^{\prime}(0) v(0) \\
& +p\left(t_{2}\right) u^{\prime}\left(t_{2}\right) v\left(t_{2}\right)-p\left(t_{1}^{+}\right) u^{\prime}\left(t_{1}^{+}\right) v\left(t_{1}^{+}\right)+\cdots \\
& +p(T) u^{\prime}(T) v(T)-p\left(t_{m}^{+}\right) u^{\prime}\left(t_{m}^{+}\right) v\left(t_{m}^{+}\right)-\int_{0}^{T}\left(p u^{\prime}\right)^{\prime} v d t \\
& -\int_{0}^{T} f(t, u) v d t+\sum_{k=1}^{m} I_{k}\left(u\left(t_{k}\right)\right) v\left(t_{k}\right) .
\end{aligned}
$$

In view of $v \in W_{p, T}^{1,2}$ and Proposition 2.1, it follows that $v\left(t_{k}\right)=v\left(t_{k}^{+}\right)$. Equation (2.3) implies

$$
\begin{array}{r}
-\sum_{k=1}^{m} \Delta\left(p\left(t_{k}\right) u^{\prime}\left(t_{k}\right)\right) v\left(t_{k}\right)+p(T) u^{\prime}(T) v(T)-p(0) u^{\prime}(0) v(0) \\
-\int_{0}^{T}\left(p u^{\prime}\right)^{\prime} v d t-\int_{0}^{T} f(t, u) v d t+\sum_{k=1}^{m} I_{k}\left(u\left(t_{k}\right)\right) v\left(t_{k}\right)=0
\end{array}
$$

holds for all $v \in W_{p, T}^{1,2}$. Without loss of generality, we assume that $v \in C_{0}^{\infty}\left(t_{k}, t_{k+1}\right)$ satisfying $v(t) \equiv 0, t \in\left[0, t_{k}\right] \cup\left[t_{k+1}, T\right]$, then substituting $v$ into (2.4) we get

$$
\int_{t_{k}}^{t_{k+1}}\left[-\left(p u^{\prime}\right)^{\prime}-f(t, u)\right] v d t=0
$$

which means

$$
-\left(p(t) u^{\prime}(t)\right)^{\prime}-f(t, u(t))=0, \quad t \in\left(t_{k}, t_{k+1}\right)
$$

Thus $u$ satisfies equation (1.1a). So (2.4) becomes

$$
\sum_{k=1}^{m}\left[I_{k}\left(u\left(t_{k}\right)\right)-\Delta\left(p\left(t_{k}\right) u^{\prime}\left(t_{k}\right)\right)\right] v\left(t_{k}\right)+p(T) u^{\prime}(T) v(T)-p(0) u^{\prime}(0) v(0)=0 .
$$

Now we will show that $u$ satisfies the impulsive condition (1.1b). If not, without loss of generality, we assume that there exists $k \in\{1,2, \ldots, m\}$ such that

$$
I_{k}\left(u\left(t_{k}\right)\right)-\Delta\left(p\left(t_{k}\right) u^{\prime}\left(t_{k}\right)\right) \neq 0 .
$$

Set $v(t)=\prod_{j=0, j \neq k}^{m+1}\left(t-t_{j}\right)=t\left(t-t_{1}\right) \cdots\left(t-t_{k-1}\right)\left(t-t_{k+1}\right) \cdots(t-T)$, combining (2.6) we get

$$
\begin{aligned}
& \sum_{k=1}^{m}\left[I_{k}\left(u\left(t_{k}\right)\right)-\Delta\left(p\left(t_{k}\right) u^{\prime}\left(t_{k}\right)\right)\right] v\left(t_{k}\right)+p(T) u^{\prime}(T) v(T)-p(0) u^{\prime}(0) v(0) \\
& \quad=\left[I_{k}\left(u\left(t_{k}\right)\right)-\Delta\left(p\left(t_{k}\right) u^{\prime}\left(t_{k}\right)\right)\right] v\left(t_{k}\right) \neq 0,
\end{aligned}
$$

which contradicts (2.5). So $u$ satisfies the impulsive condition (1.1b). From the above, we can obtain

$$
p(T) u^{\prime}(T) v(T)-p(0) u^{\prime}(0) v(0)=0
$$


Notice that $v \in W_{p, T}^{1,2}$ and $v(0)=v(T)$, so

$$
p(T) u^{\prime}(T)=p(0) u^{\prime}(0)
$$

Hence $u$ satisfies the non-separated periodic boundary condition (1.1c). Therefore, $u$ is a classical solution of problem (1.1a)-(1.1c).

Definition $2.2\left([32], P_{81}\right)$ Let $X$ be a real Banach space and $I \in C^{1}(X, R) . I$ is said to satisfy the P.S. condition on $X$ if any sequence $\left\{x_{n}\right\} \subseteq X$ for which $I\left(x_{n}\right)$ is bounded and $I^{\prime}\left(x_{n}\right) \rightarrow 0$ as $n \rightarrow \infty$ possesses a convergent subsequence in $X$.

Theorem 2.2 ([32], Theorem 4.7) Let $X$ be a Banach space and let $\Phi \in C^{1}(X, \mathbb{R})$. Assume that $X$ splits into a direct sum of closed subspaces $X=X^{-} \oplus X^{+}$with

$$
\operatorname{dim} X^{-}<\infty
$$

and

$$
\sup _{S_{R}^{-}} \Phi<\inf _{X^{+}} \Phi
$$

where $S_{R}^{-}=\left\{u \in X^{-}:\|u\|=R\right\}$. Let

$$
B_{R}^{-}=\left\{u \in X^{-}:\|u\|=R\right\}, \quad M=\left\{h \in C\left(B_{R}^{-}, X\right): h(s)=s \text { if } s \in S_{R}^{-}\right\},
$$

and

$$
c=\inf _{h \in M} \max _{s \in B_{R}^{-}} \Phi(h(s)) .
$$

Then, if $\Phi$ satisfies the P.S. condition, $c$ is a critical value of $\Phi$.

\section{Main results}

Theorem 3.1 Assume the following conditions are satisfied:

(H2) There exist $g, h \in L^{1}[0, T]$ and constant $\alpha \in[0,1)$ such that

$$
|f(t, u)| \leq g(t)|u|^{\alpha}+h(t)
$$

$$
\text { for all } u \in \mathbb{R} \text {, and a.e. } t \in[0, T] \text {. }
$$

(H3) $|u|^{-2 \alpha} \int_{0}^{T} F(t, u) d t \rightarrow-\infty$, as $|u| \rightarrow \infty$.

(H4) For any $k \in\{1,2, \ldots, m\}, I_{k}(u) u \geq 0, \forall u \in \mathbb{R}$.

Then problem (1.1a)-(1.1c) has at least one weak solution that minimizes the function J.

Proof It follows from (H2) and (2.1) that

$$
\begin{aligned}
\left|\int_{0}^{T}(F(t, u)-F(t, \bar{u})) d t\right| & =\left|\int_{0}^{T}\left(\int_{0}^{1} f(t, \bar{u}+s \tilde{u}) \tilde{u} d s\right) d t\right| \\
& \leq \int_{0}^{T} d t \int_{0}^{1}|f(t, \bar{u}+s \tilde{u})||\tilde{u}| d s
\end{aligned}
$$




$$
\begin{aligned}
& \leq \int_{0}^{T} d t \int_{0}^{1}\left[g(t)|\bar{u}+s \widetilde{u}|^{\alpha}+h(t)\right]|\widetilde{u}| d s \\
& \leq \int_{0}^{T}\left[g(t) 2^{\alpha}\left(|\bar{u}|^{\alpha}+|\widetilde{u}|^{\alpha}\right)+h(t)\right]|\widetilde{u}| d t \\
& \leq \int_{0}^{T}\left(g(t) 2^{\alpha}|\bar{u}|^{\alpha}|\widetilde{u}|+g(t) 2^{\alpha}|\widetilde{u}|^{\alpha+1}+h(t)|\widetilde{u}|\right) d t \\
& \leq 2^{\alpha}|\bar{u}|^{\alpha}\|\widetilde{u}\|_{\infty}\|g\|_{1}+2^{\alpha}\|\widetilde{u}\|_{\infty}^{\alpha+1}\|g\|_{1}+\|\widetilde{u}\|_{\infty}\|h\|_{1} \\
& \leq\|g\|_{1} 2^{\alpha}|\bar{u}|^{\alpha} C\left\|\widetilde{u}^{\prime}\right\|_{p, 2}+2^{\alpha}\|g\|_{1}\left\|\widetilde{u}^{\prime}\right\|_{p, 2}^{\alpha+1}+\|h\|_{1} C\left\|\widetilde{u}^{\prime}\right\|_{p, 2} \\
& \leq \frac{1}{4}\left\|\widetilde{u}^{\prime}\right\|_{p, 2}^{2}+C_{3}|\bar{u}|^{2 \alpha}+C_{1}\left\|\widetilde{u}^{\prime}\right\|_{p, 2}^{\alpha+1}+C_{2}\left\|\widetilde{u}^{\prime}\right\|_{p, 2} .
\end{aligned}
$$

By (H4), we have

$$
\phi(u)=\sum_{k=1}^{m} \int_{0}^{u\left(t_{k}\right)} I_{k}(s) d s \geq 0
$$

for all $u \in W_{p, T}^{1,2}$.

Therefore, we obtain

$$
\begin{aligned}
J(u) & =\frac{1}{2} \int_{0}^{T} p u^{\prime 2} d t-\int_{0}^{T} F(t, u) d t+\sum_{k=1}^{m} \int_{0}^{u\left(t_{k}\right)} I_{k}(s) d s \\
& \geq \frac{1}{2} \int_{0}^{T} p u^{\prime 2} d t-\int_{0}^{T}(F(t, u)-F(t, \bar{u})) d t-\int_{0}^{T} F(t, \bar{u}) d t \\
& \geq \frac{1}{2}\left\|\widetilde{u}^{\prime}\right\|_{p, 2}^{2}-\frac{1}{4}\left\|\widetilde{u}^{\prime}\right\|_{p, 2}^{2}-C_{3}|\bar{u}|^{2 \alpha}-C_{1}\left\|\widetilde{u}^{\prime}\right\|_{p, 2}^{\alpha+1}-C_{2}\left\|\widetilde{u}^{\prime}\right\|_{p, 2}-\int_{0}^{T} F(t, \bar{u}) d t \\
& \geq \frac{1}{4}\left\|\widetilde{u}^{\prime}\right\|_{p, 2}^{2}-|\bar{u}|^{2 \alpha}\left(|\bar{u}|^{-2 \alpha} \int_{0}^{T} F(t, \bar{u}) d t+C_{3}\right)-C_{1}\left\|\widetilde{u}^{\prime}\right\|_{p, 2}^{\alpha+1}-C_{2}\left\|\widetilde{u}^{\prime}\right\|_{p, 2}
\end{aligned}
$$

for all $u \in W_{p, T}^{1,2}$. As $\|u\| \rightarrow \infty$ if and only if $\left(\left\|\widetilde{u}^{\prime}\right\|_{p, 2}^{2}+|\bar{u}|^{2}\right)^{1 / 2} \rightarrow \infty$, (3.1) and condition (H3) imply that

$$
J(u) \rightarrow+\infty \quad \text { as }\|u\| \rightarrow \infty .
$$

Similar to the Lemma 3.1 in [34], it is easy to prove that $J$ is weakly lower semi-continues. By Theorem 1.1 in [32], $J$ has a minimum point on $W_{p, T}^{1,2}$, which is a critical point of $J$. Hence problem (1.1a)-(1.1c) has at least one weak solution.

Theorem 3.2 Assume (H2), (H3), and the following condition (H5) holds:

(H5) $\liminf _{|x| \rightarrow \infty}|x|^{\beta} \int_{0}^{x} I_{k}(s) d s>-m_{0}$ for some $\beta \in(0,2 \alpha)$ and constant $m_{0}>0$,

$$
k \in\{1,2, \ldots, m\} \text {. }
$$

Then problem (1.1a)-(1.1c) has at least one weak solution.

Proof Obviously, (H5) implies that there exists $M$ such that $\forall x \in \mathbb{R}$

$$
\int_{0}^{x} I_{k}(s) d s \geq-m_{0}|x|^{\beta}-M
$$


From Theorem 3.1, we obtain

$$
\left|\int_{0}^{T}\left(\int_{0}^{1} f(t, \bar{u}+s \tilde{u}) \tilde{u} d s\right) d t\right| \leq \frac{1}{4}\left\|\widetilde{u}^{\prime}\right\|_{p, 2}^{2}+C_{3}|\bar{u}|^{2 \alpha}+C_{1}\left\|\widetilde{u}^{\prime}\right\|_{p, 2}^{\alpha+1}+C_{2}\left\|\tilde{u}^{\prime}\right\|_{p, 2} .
$$

By (2.1) we have

$$
\begin{aligned}
\sum_{k=1}^{m}\left|u\left(t_{k}\right)\right|^{\beta} & \leq m\|u\|_{\infty}^{\beta}=m\|\bar{u}+\widetilde{u}\|_{\infty}^{\beta} \leq 2^{\beta} m\left(|\bar{u}|^{\beta}+|\widetilde{u}|^{\beta}\right) \\
& \leq 2^{\beta} m|\bar{u}|^{\beta}+2^{\beta} m C\left\|\tilde{u}^{\prime}\right\|_{p, 2}^{\beta} .
\end{aligned}
$$

Also

$$
\begin{aligned}
J(u) & =\frac{1}{2} \int_{0}^{T} p\left(u^{\prime}\right)^{2} d t-\int_{0}^{T} F(t, u) d t+\sum_{k=1}^{m} \int_{0}^{u\left(t_{k}\right)} I_{k}(s) d s \\
& =\frac{1}{2} \int_{0}^{T} p\left(u^{\prime}\right)^{2} d t-\int_{0}^{T}(F(t, u)-F(t, \bar{u})) d t-\int_{0}^{T} F(t, \bar{u}) d t+\sum_{k=1}^{m} \int_{0}^{u\left(t_{k}\right)} I_{k}(s) d s .
\end{aligned}
$$

Substituting (3.2) and (3.3) into the above equation, we have

$$
\begin{aligned}
J(u) \geq & \frac{1}{2}\left\|\tilde{u}^{\prime}\right\|_{p, 2}^{2}-\frac{1}{4}\left\|\tilde{u}^{\prime}\right\|_{p, 2}^{2}-C_{3}|\bar{u}|^{2 \alpha}-C_{1}\left\|\tilde{u}^{\prime}\right\|_{p, 2}^{\alpha+1}-C_{2}\left\|\tilde{u}^{\prime}\right\|_{p, 2} \\
& -\sum_{k=1}^{m} m_{0}\left|u\left(t_{k}\right)\right|^{\beta}-m M-\int_{0}^{T} F(t, \bar{u}) d t \\
= & \frac{1}{4}\left\|\tilde{u}^{\prime}\right\|_{p, 2}^{2}-C_{3}|\bar{u}|^{2 \alpha}-C_{1}\left\|\tilde{u}^{\prime}\right\|_{p, 2}^{\alpha+1}-C_{2}\left\|\tilde{u}^{\prime}\right\|_{p, 2}-C_{6}|\bar{u}|^{\beta}-\left\|\tilde{u}^{\prime}\right\|_{p, 2}^{\beta} \\
& -\int_{0}^{T} F(t, \bar{u}) d t+C_{7} \\
\geq & \frac{1}{4}\left\|\tilde{u}^{\prime}\right\|_{p, 2}^{2}-|\bar{u}|^{2 \alpha}\left[|\bar{u}|^{-2 \alpha} \int_{0}^{T} F(t, \bar{u}) d t+\frac{C_{6}|\bar{u}|^{\beta}}{|\bar{u}|^{2 \alpha}}+C_{3}\right] \\
& -C_{1}\left\|\tilde{u}^{\prime}\right\|_{p, 2}^{\alpha+1}-C_{2}\left\|\tilde{u}^{\prime}\right\|_{p, 2}+C_{7} .
\end{aligned}
$$

Since $\beta \in(0,2 \alpha)$, the above inequality and (H3) imply that

$$
J(u) \rightarrow+\infty, \quad \text { as }\|u\| \rightarrow \infty
$$

So $J$ has a minimum point on $W_{p, T}^{1,2}$, which is a critical point of $J$. Hence problem (1.1a)(1.1c) has at least one solution.

Theorem 3.3 Suppose that the condition (H2) of Theorem 3.1 hold. Assume the following: (H6) There exist $a_{k}, b_{k}>0$, and $\gamma \in(0, \alpha)$ such that

$$
\left|I_{k}(s)\right| \leq a_{k}+b_{k}|s|^{\gamma}
$$

for every $s \in \mathbb{R}, k \in\{1,2, \ldots, m\}$. 
(H7) For any $k \in\{1,2, \ldots, m\}, I_{k}(s) s \leq 0, \forall s \in \mathbb{R}$.

(H8) $|u|^{-2 \alpha} \int_{0}^{T} F(t, u) d t \rightarrow+\infty$, as $|u| \rightarrow+\infty$.

Then problem (1.1a)-(1.1c) has at least one weak solution.

First of all, we prove the following lemma.

Lemma 3.1 Suppose that the conditions of Theorem 3.3 hold, then J satisfies the P.S. condition.

Proof Let $\left\{u_{n}\right\} \subset W_{p, T}^{1,2}$ be a P.S. sequence for $J$, that is, $\left\{J\left(u_{n}\right)\right\}$ is bounded, $J^{\prime}\left(u_{n}\right) \rightarrow 0$ as $n \rightarrow \infty$. It follows from $(\mathrm{H} 2)$ and (2.1) that

$$
\begin{aligned}
& \left|\int_{0}^{T} f\left(t, u_{n}\right) \tilde{u}_{n} d t\right| \leq \int_{0}^{T}\left|f\left(t, u_{n}\right)\right|\left|\tilde{u}_{n}\right| d t \\
& \leq \int_{0}^{T}\left(g(t)\left|u_{n}\right|^{\alpha}+h(t)\right)\left|\widetilde{u}_{n}\right| d t \\
& \leq \int_{0}^{T} g(t)\left|u_{n}\right|^{\alpha}\left|\tilde{u}_{n}\right| d t+\int_{0}^{T} h(t)\left|\tilde{u}_{n}\right| d t \\
& \leq\left\|u_{n}\right\|_{\infty}^{\alpha}\left\|\tilde{u}_{n}\right\|_{\infty}\|g\|_{1}+\left\|\tilde{u}_{n}\right\|_{\infty}\|h\|_{1} \\
& \leq\left\|\bar{u}_{n}+\tilde{u}_{n}\right\|_{\infty}^{\alpha}\left\|\tilde{u}_{n}\right\|_{\infty}\|g\|_{1}+\left\|\tilde{u}_{n}\right\|_{\infty}\|h\|_{1} \\
& \leq 2^{\alpha}\left(|\bar{u}|^{\alpha}+\left\|\tilde{u}_{n}\right\|_{\infty}^{\alpha}\right)\left\|\tilde{u}_{n}\right\|_{\infty}\|g\|_{1}+\left\|\tilde{u}_{n}\right\|_{\infty}\|h\|_{1} \\
& \leq 2^{\alpha}|\bar{u}|^{\alpha}\left\|\tilde{u}_{n}\right\|_{\infty}\|g\|_{1}+2^{\alpha}\left\|\tilde{u}_{n}\right\|_{\infty}^{\alpha+1}\|g\|_{1}+\left\|\tilde{u}_{n}\right\|_{\infty}\|h\|_{1} \\
& \leq 2^{\alpha}\left|\bar{u}_{n}\right|^{\alpha}\|g\|_{1} C\left\|\tilde{u}_{n}^{\prime}\right\|_{p, 2}+2^{\alpha}\|g\|_{1} C^{\alpha+1}\left\|\tilde{u}_{n}^{\prime}\right\|_{p, 2}^{\alpha+1}+C_{2} C\left\|\tilde{u}_{n}^{\prime}\right\|_{p, 2} \\
& \leq \frac{1}{4}\left\|\widetilde{u}_{n}^{\prime}\right\|_{p, 2}^{2}+C_{4}\left|\bar{u}_{n}\right|^{2 \alpha}+C_{5}\left\|\widetilde{u}_{n}^{\prime}\right\|_{p, 2}^{\alpha+1}+C_{6}\left\|\widetilde{u}_{n}^{\prime}\right\|_{p, 2} .
\end{aligned}
$$

Therefore

$$
\begin{aligned}
\left\langle J^{\prime}\left(u_{n}\right), \tilde{u}_{n}\right\rangle= & \int_{0}^{T} p u_{n}^{\prime} \widetilde{u}_{n}^{\prime} d t-\int_{0}^{T} f\left(t, u_{n}\right) \widetilde{u}_{n} d t+\sum_{k=1}^{m} I_{k}\left(u_{n}\left(t_{k}\right)\right) \widetilde{u}_{n}\left(t_{k}\right) \\
\geq & \frac{3}{4}\left\|\widetilde{u}_{n}^{\prime}\right\|_{p, 2}^{2}-C_{4}\left|\bar{u}_{n}\right|^{2 \alpha}-C_{5}\left\|\widetilde{u}_{n}^{\prime}\right\|_{p, 2}^{\alpha+1}-C_{6}\left\|\widetilde{u}_{n}^{\prime}\right\|_{p, 2}+\sum_{k=1}^{m} I_{k}\left(u_{n}\left(t_{k}\right)\right) \widetilde{u}_{n}\left(t_{k}\right) \\
\geq & \frac{3}{4}\left\|\widetilde{u}_{n}^{\prime}\right\|_{p, 2}^{2}-C_{4}\left|\bar{u}_{n}\right|^{2 \alpha}-C_{5}\left\|\tilde{u}_{n}^{\prime}\right\|_{p, 2}^{\alpha+1}-C_{6}\left\|\widetilde{u}_{n}^{\prime}\right\|_{p, 2} \\
& -\sum_{k=1}^{m}\left(a_{k}+b_{k}\left|u_{n}\left(t_{k}\right)\right|^{\gamma}\right)\left|\widetilde{u}_{n}\left(t_{k}\right)\right|
\end{aligned}
$$

Let $a=\max \left\{a_{i}\right\}, b=\max \left\{b_{i}\right\}$, one has

$$
\begin{aligned}
\left\langle J^{\prime}\left(u_{n}\right), \tilde{u}_{n}\right\rangle \geq & \frac{3}{4}\left\|\tilde{u}_{n}^{\prime}\right\|_{p, 2}^{2}-C_{4}\left|\bar{u}_{n}\right|^{2 \alpha}-C_{5}\left\|\widetilde{u}_{n}^{\prime}\right\|_{p, 2}^{\alpha+1}-C_{6}\left\|\widetilde{u}_{n}^{\prime}\right\|_{p, 2} \\
& \quad-a m\left\|\tilde{u}_{n}\right\|_{\infty}-b \sum_{k=1}^{m} 2\left(\left|\bar{u}_{n}\right|^{\gamma}+\left\|\widetilde{u}_{n}\right\|_{\infty}^{\gamma}\right)\left\|\widetilde{u}_{n}\right\|_{\infty}
\end{aligned}
$$




$$
\begin{aligned}
\geq & \frac{3}{4}\left\|\widetilde{u}_{n}^{\prime}\right\|_{p, 2}^{2}-C_{4}\left|\bar{u}_{n}\right|^{2 \alpha}-C_{5}\left\|\widetilde{u}_{n}^{\prime}\right\|_{p, 2}^{\alpha+1}-C_{6}\left\|\widetilde{u}_{n}^{\prime}\right\|_{p, 2}-a m C\left\|\widetilde{u}_{n}^{\prime}\right\|_{p, 2} \\
& -2 m b\left|\bar{u}_{n}\right|^{\gamma}\left\|\tilde{u}_{n}\right\|_{\infty}-2 b m C\left\|\widetilde{u}_{n}^{\prime}\right\|_{p, 2}^{\gamma+1} .
\end{aligned}
$$

By Yang inequality and (2.1), the following inequality holds:

$$
\left|\bar{u}_{n}\right|^{\gamma}\left\|\widetilde{u}_{n}\right\|_{\infty} \leq \frac{\left(\left|\bar{u}_{n}\right|^{\gamma}\right)^{2 \alpha / \gamma}}{2 \alpha / \gamma}+\frac{\left\|\widetilde{u}_{n}\right\|_{\infty}^{\frac{2 \gamma}{2 \alpha-\gamma}}}{\frac{2 \alpha}{2 \alpha-\gamma}} .
$$

From the above, we have

$$
\begin{gathered}
\left\|\widetilde{u}_{n}\right\| \geq\left\langle J^{\prime}\left(u_{n}\right), \widetilde{u}_{n}\right\rangle \geq \frac{3}{4}\left\|\widetilde{u}_{n}^{\prime}\right\|_{p, 2}^{2}-C_{4}\left|\bar{u}_{n}\right|^{2 \alpha}-C_{5}\left\|\widetilde{u}_{n}^{\prime}\right\|_{p, 2}^{\alpha+1}-C_{6}\left\|\widetilde{u}_{n}^{\prime}\right\|_{p, 2} \\
-a m C\left\|\widetilde{u}_{n}^{\prime}\right\|_{p, 2}-2 b m\left\|\widetilde{u}_{n}^{\prime}\right\|_{p, 2}^{\gamma+1}-C_{7}\left|\bar{u}_{n}\right|^{2 \alpha}-C_{8}\left\|\widetilde{u}_{n}^{\prime}\right\|_{p, 2}^{\frac{2 \alpha}{2 \alpha-\gamma}}
\end{gathered}
$$

On the other hand, in view of (2.2), we have

$$
\begin{aligned}
\left\|\widetilde{u}_{n}^{\prime}\right\|_{p, 2}^{2} & \leq\left\|\widetilde{u}_{n}\right\|^{2}=\int_{0}^{T} p\left(\widetilde{u}_{n}^{\prime}\right)^{2} d t+\int_{0}^{T}\left(\widetilde{u}_{n}\right)^{2} d t \\
& \leq \int_{0}^{T} p\left(\widetilde{u}_{n}^{\prime}\right)^{2} d t+\frac{1}{\mu_{1}} \int_{0}^{T} p\left(\widetilde{u}_{n}^{\prime}\right)^{2} d t=\left(1+\frac{1}{\mu_{1}}\right)\left\|\widetilde{u}_{n}^{\prime}\right\|_{p, 2}^{2} .
\end{aligned}
$$

So,

$$
C\left\|\widetilde{u}_{n}^{\prime}\right\|_{p, 2} \geq \frac{3}{4}\left\|\widetilde{u}_{n}^{\prime}\right\|_{p, 2}^{2}-\left(C_{4}+C_{7}\right)\left|\bar{u}_{n}\right|^{2 \alpha}-C_{5}\left\|\widetilde{u}_{n}^{\prime}\right\|_{p, 2}^{\alpha+1}-a m C\left\|\widetilde{u}_{n}^{\prime}\right\|_{p, 2}
$$

which means

$$
C_{9}\left|\bar{u}_{n}\right|^{\alpha} \geq\left\|\widetilde{u}_{n}^{\prime}\right\|_{p, 2}-C_{10}
$$

Like in the proof of Theorem 3.1, we have

$$
\left|\int_{0}^{T}\left[F\left(t, u_{n}\right)-F\left(t, \bar{u}_{n}\right)\right] d t\right| \leq \frac{1}{4}\left\|\widetilde{u}_{n}^{\prime}\right\|_{p, 2}^{2}+C_{4}\left|\bar{u}_{n}\right|^{2 \alpha}+C_{5}\left\|\widetilde{u}_{n}^{\prime}\right\|_{p, 2}^{\alpha+1}+C_{6}\left\|\widetilde{u}_{n}^{\prime}\right\|_{p, 2} .
$$

By the boundedness of $\left\{J\left(u_{n}\right)\right\}$, (H7), (3.5), and (3.6), there exists a constant $C$ such that

$$
\begin{aligned}
C & \leq J\left(u_{n}\right)=\frac{1}{2} \int_{0}^{T} p\left(u_{n}^{\prime}\right)^{2} d t-\int_{0}^{T} F\left(t, u_{n}\right) d t+\sum_{k=0}^{m} \int_{0}^{u_{n}\left(t_{k}\right)} I_{k}(s) d s \\
& \leq \frac{1}{2}\left\|\widetilde{u}_{n}^{\prime}\right\|_{p, 2}^{2}-\int_{0}^{T}\left[F\left(t, u_{n}\right)-F\left(t, \bar{u}_{n}\right)\right] d t-\int_{0}^{T} F\left(t, \bar{u}_{n}\right) d t \\
& \leq \frac{3}{4}\left\|\widetilde{u}_{n}^{\prime}\right\|_{p, 2}^{2}+C_{4}\left|\bar{u}_{n}\right|^{2 \alpha}+C_{5}\left\|\widetilde{u}_{n}^{\prime}\right\|_{p, 2}^{\alpha+1}+C_{6}\left\|\widetilde{u}_{n}^{\prime}\right\|_{p, 2}-\int_{0}^{T} F(t, \bar{u}) d t \\
& \leq\left|\bar{u}_{n}\right|^{2 \alpha}\left(-\left|\bar{u}_{n}\right|^{2 \alpha} \int_{0}^{T} F\left(t, \bar{u}_{n}\right) d t+C_{11}\right) .
\end{aligned}
$$


It follows from (3.7) and (H8) that $\left\{\left|\bar{u}_{n}\right|\right\}$ is bounded. Hence $\left\{u_{n}\right\}$ is bounded in $W_{p, T}^{1,2}$ by (3.5). Therefore, there exists a subsequence of $u_{n}$ (for simplicity denoted again by $\left\{u_{n}\right\}$ ) such that

$$
u_{n} \rightarrow u \quad \text { in } W_{p, T}^{1,2}
$$

By Proposition 2.1, one has

$$
u_{n} \rightarrow u \quad \text { in } C[0, T]
$$

On the other hand, we have

$$
\begin{aligned}
\left\langle J^{\prime}\left(u_{n}\right)-J^{\prime}(u), u_{n}-u\right\rangle= & \int_{0}^{T} p\left(u_{n}^{\prime}-u^{\prime}\right)^{2} d t-\int_{0}^{T}\left(f\left(t, u_{n}\right)-f(t, u)\right)\left(u_{n}-u\right) d t \\
& +\sum_{k=1}^{m}\left(I_{k}\left(u_{n}\left(t_{k}\right)\right)-I_{k}\left(u\left(t_{k}\right)\right)\right)\left(u_{n}\left(t_{k}\right)-u\left(t_{k}\right)\right) .
\end{aligned}
$$

From (3.8)-(3.10), (H), and the continuity of $I_{k}$, it follows that $u_{n} \rightarrow u$ in $W_{p, T}^{1,2}$. Thus, $J$ satisfies the P.S. condition.

Now, we prove Theorem 3.3.

Proof From Section 2, we know $W_{p, T}^{1,2}=\bar{W} \oplus \widetilde{W}$. We show that

$$
J(u) \rightarrow+\infty \quad \text { as } u \in \widetilde{W},\|u\| \rightarrow \infty
$$

If $u \in \widetilde{W}$, then $\bar{u}=0$. Like in the proof of Theorem 3.1, we have

$$
\left|\int_{0}^{T}[F(t, u)-F(t, 0)] d t\right| \leq \frac{1}{4}\left\|u^{\prime}\right\|_{p, 2}^{2}+C_{5}\left\|u^{\prime}\right\|_{p, 2}^{\alpha+1}+C_{6}\left\|u^{\prime}\right\|_{p, 2} .
$$

By (H6) and (2.1) we find

$$
\begin{aligned}
\psi(u) & :=\sum_{k=1}^{m} \int_{0}^{u\left(t_{k}\right)} I_{k}(s) d s \leq \sum_{k=1}^{m} \int_{0}^{u\left(t_{k}\right)}\left(a_{k}+b_{k}|s|^{\gamma}\right) d s \\
& \leq a m\|u\|_{\infty}+b \sum_{k=1}^{m}\|u\|_{\infty}^{\gamma+1} \\
& \leq a m C\left\|u^{\prime}\right\|_{p, 2}+b \sum_{k=1}^{m}\left\|u^{\prime}\right\|_{p, 2}^{\gamma+1},
\end{aligned}
$$

for all $u \in \widetilde{W}$. It follows from (3.12) and (3.13) that

$$
\begin{aligned}
J(u) & =\frac{1}{2} \int_{0}^{T} p u^{\prime 2} d t-\int_{0}^{T}[F(t, u)-F(t, 0)] d t-\int_{0}^{T} F(t, 0) d t+\psi(u) \\
& \geq \frac{1}{4}\left\|u^{\prime}\right\|_{p, 2}^{2}-C_{5}\left\|u^{\prime}\right\|_{p, 2}^{\alpha+1}-C_{6}\left\|u^{\prime}\right\|_{p, 2}-C_{7}\left\|u^{\prime}\right\|_{p, 2}-C_{8} \sum_{k=1}^{m}\left\|u^{\prime}\right\|_{p, 2}^{\gamma+1}
\end{aligned}
$$

for all $u \in \widetilde{W}$. Hence, (3.11) follows from (3.4) and (3.14). 
On the other hand, by (H7), we obtain

$$
\psi(u) \leq 0
$$

for all $u \in W_{p, T}^{1,2}$. Hence, by (3.15) and (H8), one has

$$
\begin{aligned}
J(u) & =-\int_{0}^{T} F(t, u) d t+\psi(u) \\
& \leq|u|^{2 \alpha}\left[-|u|^{-2 \alpha} \int_{0}^{T} F(t, u) d t\right] \rightarrow-\infty
\end{aligned}
$$

as $|u| \rightarrow \infty$ in $\mathbb{R}$. By Theorem 2.2 and Lemma 3.1, problem (1.1a)-(1.1c) has at least one weak solution.

\section{Competing interests}

The authors declare that they have no competing interests.

\section{Authors' contributions}

$\mathrm{RL}$ carried out the main part of this article, WZ corrected the manuscript and brought forward many suggestions on this article. All authors have read and approved the final manuscript.

\section{Acknowledgements}

The authors would like to thank the referees for their pertinent comments and valuable suggestions. This work was supported by the National Natural Science Foundation of China (11001274).

Received: 18 March 2016 Accepted: 27 May 2016 Published online: 03 June 2016

\section{References}

1. Geoge, RK, Nandakumaran, AK, Arapostathis, A: A note on controllability of impulsive systems. J. Math. Anal. Appl. 241, 276-283 (2000)

2. Jiang, G, Lu, Q: Impulsive state feedback control of a predator-prey model. J. Comput. Appl. Math. 200, 193-207 (2007)

3. Nenov, S: Impulsive controllability and optimization problems in population dynamics. Nonlinear Anal., Theory Methods Appl. 36, 881-890 (1999)

4. D'Onofrio, A: On impulse vaccination strategy in the SIR epidemic model with vertical transmission. Appl. Math. Lett. $18,729-732(2005)$

5. Choisy, M, Guegan, JF, Rohani, P: Dynamics of infectious diseases and pulse vaccination: teasing apart the embedded resonance effects. Physica D 223, 26-35 (2006)

6. Gao, S, Chen, L, Nieto, JJ, Torres, A: Analysis of a delayed epidemic model with pulse vaccination and saturation incidence. Vaccine 24, 6037-6045 (2006)

7. Prado, A: Bi-impulsive control to build a satellite constellation. Nonlinear Dyn. Syst. Theory 5, 169-175 (2005)

8. Qian, D, Li, X: Periodic solutions for ordinary differential equations with sublinear impulsive effects. J. Math. Anal. Appl. 303, 288-303 (2005)

9. Chen, L, Sun, J: Nonlinear boundary value problem for first order impulsive functional differential equations. J. Math. Anal. Appl. 318, 726-741 (2006)

10. Liang, R, Shen, J: Periodic boundary value problem for the first order impulsive functional differential equations. J. Comput. Appl. Math. 202, 498-510 (2007)

11. Liang, R, Shen, J: Periodic boundary value problem for second-order impulsive functional differential equations. Appl. Math. Lett. 193, 560-571 (2007)

12. Liang, R, Shen, J: Periodic boundary value problem for the first order functional differential equations with impulses. Appl. Math. J. Chin. Univ. Ser. B 24, 27-35 (2009)

13. Liang, R, Shen, J: Eigenvalue criteria for existence of positive solutions of impulsive differential equations with non-separated boundary conditions. Bound. Value Probl. 2013, 3 (2013)

14. Chen, L, Tisdel, C, Yuan, R: On the solvability of periodic boundary value problems with impulses. J. Math. Anal. Appl. 331, 233-244 (2007)

15. Chu, J, Nieto, J: Impulsive periodic solution of first order singular differential equations. Bull. Lond. Math. Soc. 40 143-150 (2008)

16. Lakshmikantham, V, Bainov, DD, Simeonov, PS: Theory of Impulsive Differential Equations. World Scientific, Singapore (1989)

17. Samoilenko, AM, Perestyuk, NA: Impulsive Differential Equations. World Scientific, Singapore (1995)

18. Wang, W, Yang, X: Multiple solutions of boundary-value problems for impulsive differential equations. Math. Methods Appl. Sci. 34, 1649-1657 (2011)

19. Atici, F, Guseinov, G: On the existence of positive solutions for nonlinear differential equations with periodic boundary conditions. J. Comput. Appl. Math. 132, 341-356 (2001) 
20. Graef, J, Kong, J: Existence results for nonlinear periodic boundary-value problems. Proc. Edinb. Math. Soc. 52, 79-95 (2009)

21. Han, Z: Solutions of periodic boundary value problems for second-order nonlinear differential equations via variational methods. Appl. Math. Comput. 217, 6516-6525 (2011)

22. Hao, $X, L i u, L, W u, Y$ : Existence and multiplicity results for nonlinear periodic boundary value problems. Nonlinear Anal. TMA 72, 3635-3642 (2010)

23. Tang, C: Periodic solutions for nonautonomous second order systems with sublinear nonlinearity. Proc. Am. Math. Soc. 126, 3263-3270 (1998)

24. Huseynov, A: On the sign of Green's function for an impulsive differential equation with periodic boundary conditions. Appl. Math. Comput. 208, 197-205 (2009)

25. Huseynov, A: Positive solutions of a nonlinear impulsive equation with periodic boundary conditions. Appl. Math Comput. 217, 247-259 (2010)

26. Xiao, J, Nieto, J, Luo, Z: Multiplicity of solutions for nonlinear second order impulsive differential equations with linear derivative dependence via variational methods. Commun. Nonlinear Sci. Numer. Simul. 17, 426-432 (2012)

27. Dai, B, Zhang, D: The existence and multiplicity of solutions for second-order impulsive differential equations on the half-line. Results Math. 63, 135-149 (2013)

28. Sun, J, Chen, H: Multiplicity of solutions for a class of impulsive differential equations with Dirichlet boundary conditions via variant fountain theorems. Nonlinear Anal., Real World Appl. 11, 4062-4071 (2010)

29. Sun, J, Chen, H: Variational method to the impulsive equation with Neumann boundary conditions. Bound. Value Probl. 2009, 316812 (2009)

30. Chen, $\mathrm{H}, \mathrm{Li}$, J: Variational approach to impulsive differential equations with Dirichlet boundary conditions. Bound. Value Probl. 2010, 325415 (2010)

31. Liang, R: Existence of solutions for impulsive Dirichlet problems with the parameter inequality reverse. Math. Methods Appl. Sci. 36, 1929-1936 (2013)

32. Mawhin, J, Willem, M: Critical Point Theory and Hamiltonian Systems. Springer, Berlin (1989)

33. Ravinowitz, P: Minimax Methods in Critical Point Theory with Applications to Differential Equations. Am. Math. Soc., Providence (1986)

34. Zhou, J, Li, Y: Existence of solutions for a class of second-order Hamiltonian systems with impulsive effects. Nonlinear Anal. TMA 72, 1594-1603 (2010)

35. Agarwal, R, Bhaskar, T, Perera, K: Some results for impulsive problems via Morse theory. J. Math. Anal. Appl. 409(2), 752-759 (2014)

36. Bhaskar, T, Perera, K: On some elliptic interface problems with nonhomogeneous jump conditions. Adv. Nonlinear Anal. 2, 195-211 (2013)

\section{Submit your manuscript to a SpringerOpen ${ }^{\circ}$ journal and benefit from:}

- Convenient online submission

- Rigorous peer review

- Immediate publication on acceptance

- Open access: articles freely available online

High visibility within the field

- Retaining the copyright to your article 